\title{
Pharmaceutical care - impact on quality of life in patients with type 2 diabetes: a review
}

This article was published in the following Dove Press journal:

Clinical Audit

6 March 2013

Number of times this article has been viewed

\author{
Ines Krass' \\ Teerapon Dhippayom ${ }^{2}$ \\ 'Faculty of Pharmacy, University \\ of Sydney, Sydney, NSW, Australia; \\ ${ }^{2}$ Faculty of Pharmaceutical Sciences, \\ Naresuan University, Phitsanulok, \\ Thailand
}

Correspondence: Ines Krass Faculty of Pharmacy, University of Sydney, Science Road A I5, Camperdown, Sydney, NSW 2006, Australia

Tel +6I 2 935। 3507

Fax +6I 2 935। 439|

Email ines.krass@sydney.edu.au
Abstract: Type 2 diabetes mellitus (T2DM), a chronic disorder now reaching epidemic proportions, imposes a huge burden on individuals and health care systems. In recent years, pharmacists, highly trained health care professionals with expertise in medicines, have sought to develop an expanded role in diabetes care. Evidence suggests that pharmaceutical care produces improvements in glycemic control; however, little is known about its impact on humanistic outcomes such as health-related quality of life (HRQoL). This review aimed to address this gap. A systematic search was conducted of English language articles published from 1996 to January, 2013 in Cochrane databases of systematic reviews and clinical trials, CINAHL, Embase, MEDLINE, PubMed, International Pharmaceutical Abstracts, PsycINFO, and Web of Science databases to identify relevant original research articles and reviews linking pharmaceutical care, T2DM, and HRQoL. The quality of selected articles was assessed using a modified version of the Downs and Black checklist. Of a total of 122 articles addressing pharmaceutical care in T2DM, 17 articles were suitable for inclusion: 12 studies used generic HRQoL instruments, six used diabetes-specific HRQoL scales, and one study used both. Because of the different scales used and the level of detail, it is difficult to compare between studies. The results provide some preliminary evidence that pharmaceutical care in T2DM can have a positive impact on HRQoL, with the evidence pointing to a greater effect on mental rather than physical health; however, these findings are inconclusive. The mean quality score for the 13 studies included in the quality rating was $0.63 \pm 0.11$ (range $0.40-0.76$ ), which is classified as only fair. Future studies should use robust research designs to bolster the evidence for the impact of pharmaceutical care on HRQoL using both generic and disease-specific measures.

Keywords: health-related quality of life, pharmacist intervention, disease management, health outcomes, generic measures, disease-specific measures

\section{Introduction}

Type 2 diabetes mellitus (T2DM) is a chronic progressive metabolic disorder which is now reaching epidemic proportions in both developed and developing countries. In 2010 , an estimated $6.4 \%$ or 285 million adults were affected globally, and this is predicted to increase to $7.7 \%$ (439 million adults) by $2030 .{ }^{1} \mathrm{~T} 2 \mathrm{DM}$ imposes a huge burden on individuals, communities, and health care systems, with estimated direct costs accounting for $2.5 \%-15 \%$ of health care budgets, depending on prevalence and available treatment options. ${ }^{2}$ The morbidity and mortality associated with T2DM result from complications associated with the duration and severity of disease and degree of disease control. These include coronary heart disease, stroke, blindness, and kidney failure. ${ }^{3}$ 
In the UK Prospective Diabetes Study trial, strict control of glycemia and cardiovascular risk factors, including blood pressure and lipids, were shown to reduce the risk and delay the onset of complications and bring about improvements in overall quality of life (QoL) for the patient. ${ }^{4-6}$ Translation of evidence into practice and delivering optimal care represents a significant challenge to health care systems across the globe. Because of the nature of T2DM and the need for patients to understand and take control of their lifestyle to reduce their health risk, patients need intensive chronic disease management and ongoing support. From a systems perspective, limited access to diabetes health care professionals as a result of personnel shortages, misdistribution of services, and lack of systems to support chronic disease management with continuity of care is well recognized as a barrier to achieving optimal health outcomes for the diabetes population. ${ }^{7,8}$ Indeed, evidence suggests that control of glycemia and other cardiovascular risk factors among many people with T2DM remains suboptimal. ${ }^{9-11}$ Thus, there is a need to increase the capacity to deliver more intensive diabetes management and support through innovation in models of care delivery and by leveraging existing health care resources in the community to enhance access to care for the burgeoning populations with T2DM.

In recent years, pharmacists, highly trained health care professionals with expertise in medicines, have sought to develop an expanded role in diabetes care to meet the needs of this patient population. There are compelling arguments that support this expanded involvement. Pharmacists in the community, through regular and less formal contact than that with doctors, are able to build strong relationships with patients and become a reliable source of information. Pharmacists in both community and clinic settings can also have ongoing relationships with other health care providers and can serve as the "bridge" between health care providers and the patients, thus ensuring continuity of care. In addition, as medications play a key role in preventing the complications of T2DM, ensuring their effectiveness through monitoring and supporting adherence and screening for drug related problems is critical to achieving improved health outcomes. Expansion of diabetes care roles for pharmacists also aligns well with a paradigm shift from a focus on the drug product to a more patient-centered practice orientation, labeled by Hepler and Strand as "pharmaceutical care." 12 Defined as "the responsible provision of drug therapy for the purpose of achieving definite outcomes that improve a patient's QoL," pharmaceutical care requires the cooperation of the pharmacist with the patient and other professionals in "designing, implementing, and monitoring a therapeutic plan" with the pharmacist directly responsible to the patient. ${ }^{12}$ A growing body of literature has reported improvements in glycemic control and/or changes in cardiovascular risk factors when pharmacists provide pharmaceutical care and diabetes disease state management services in research situations in both the clinic and community pharmacy settings. ${ }^{13-15}$ A meta-analysis of 16 studies evaluating the impact of pharmacist interventions in diabetes management report a mean difference in reduction of hemoglobin $\mathrm{A} 1 \mathrm{c}\left(\mathrm{HbA}_{1 \mathrm{c}}\right)$ of $0.62 \% \pm 0.29 \%$ between intervention and control subjects in favor of the pharmacist intervention. ${ }^{13}$ However, any comprehensive evaluation of a pharmaceutical service requires the economic, clinical, and humanistic outcomes (ECHO) approach, which determines its value as a combination of traditional clinical-based outcomes with more contemporary measures of economic efficiency and quality. ${ }^{16}$ The most important humanistic outcome, which is a key focus of pharmaceutical care, is health-related QoL (HRQoL).

In general, people with T2DM have poorer QoL than those without a chronic disease. Day to day, to keep blood glucose levels within a normal range, they must take medication, carefully control their diet and physical activity, and undertake regular self-monitoring of blood glucose. Long term, this can exact a heavy psychosocial toll, which in turn may affect self-management behavior and, ultimately, long-term glycemic control, the risk of developing long-term complications, and QoL. ${ }^{17}$

\section{HRQoL}

QoL is, therefore, now well accepted as one of the important goals and outcomes in the treatment of diabetes. ${ }^{18}$ Although definitions vary, the most widely used definition describes QoL as "an individual's perception of their position in life in the context of culture and value systems in which they live, and in relation to their goals, expectations, standards, and concerns." ${ }^{19}$ Since QoL comprises all aspects of life, the term HRQoL has gradually been adopted in most of the health science literature because there are aspects of life that are not generally considered as "health." ${ }^{20}$ In general, HRQoL is a multidimensional construct of an individual's subjective appraisal of health and wellbeing involving physical, psychological, and social functioning. ${ }^{21}$

The measurement of HRQoL can be categorized as generic and disease specific. Generic instruments are used for the general population in a variety of health conditions and diseases. They can be employed when patients have multiple chronic conditions and can be used to compare HRQoL 
across diseases. ${ }^{21}$ Disease-specific instruments, on the other hand, are mainly designed to be more responsive to detecting subtle changes in QoL that are important to a specific disease. A systematic review by El Achhab et al showed that there were 16 instruments that have been developed to measure HRQoL in patients with diabetes, although not all of them were formally assessed for responsiveness. The items listed in those tools ranged from seven to 67 items. ${ }^{22}$

Another systematic review of HRQoL measures used in diabetes research, showed that only three instruments among the ten most frequently used instruments may actually be considered as measures of QoL: the Diabetes QoL (DQOL), the Audit of Diabetes Dependent QoL (ADDQoL), and the generic World Health Organization QoL (WHOQOL). ${ }^{23}$

The DQOL was the first diabetes-specific QoL measure, and was originally designed for use in the Diabetes Control and Complications Trial. ${ }^{24}$ It consists of 46 items, with each item scored on a five-point Likert scale. The main limitation of DQOL is that there is no scope within the measure for an individual to indicate that a given aspect of life is not applicable to them and all items are treated as equally important to each respondent. The ADDQoL was designed to address this limitation and it provides an individualized measure of the impact of diabetes on QoL. ${ }^{25}$ Comprised of a 19-item scale, it has been translated into more than 23 languages. The WHOQOL-100 and its short form, WHOQOL-BREF, were developed through international consensus as generic measurements of QoL. ${ }^{26}$ Although the WHOQOL is not diabetes-specific measure, it is relevant to patients with diabetes and has been extensively used in diabetes studies.

Other generic instruments used to measure QoL in diabetes research include the Short Form 36 (SF-36) and the EuroQoL-5 Dimension (EQ-5D), although some have suggested that these tools more accurately measure health status rather than QoL..$^{23}$ The SF-36, which is the most widely used general health status instrument, includes eight multiple-item scales that cover the dimensions of: (1) physical functioning; (2) role-physical; (3) pain; (4) general health; (5) vitality; (6) social functioning; (7) role-emotional; and (8) mental health. ${ }^{27}$ Changes in SF-36 scores of less than four are considered small, four to ten are considered moderate, and more than ten are considered large. ${ }^{28}$ The EQ-5D measures health status in terms of five dimensions: (1) mobility; (2) self-care; (3) usual activities; (4) pain/discomfort; and (5) anxiety/ depression. A body of literature has demonstrated that the EQ-5D is simple to administer and has demonstrated psychometric properties comparable with other instruments. ${ }^{29,30}$
Overall, HRQoL comprises a wide range of dimensions, and the current available measures - both generic and diabetes specific - cannot cover all of these. In addition, there is no QoL instrument that has been specifically designed for use in the evaluation of pharmaceutical care interventions. ${ }^{31}$ Existing HRQoL measures are not necessarily sensitive to the impacts of the elements of pharmaceutical care.

While all trials of pharmaceutical care and pharmacist disease management diabetes services have included clinical outcomes measures, few have focused on humanistic outcomes such as HRQoL. The aim of this review was to identify and assess the impact of pharmaceutical care on HRQoL in patients with T2DM. For the purposes of this review, pharmaceutical care was operationally defined as any intervention delivered in any health care setting by a pharmacist to patients with T2DM to improve disease management and health outcomes.

\section{Methods}

A search was conducted of English language articles published from 1996 to January 2013 in Cochrane databases of systematic reviews and clinical trials, CINAHL, Embase, MEDLINE, PubMed, International Pharmaceutical Abstracts, PsycINFO, and Web of Science databases to identify relevant original research articles and reviews linking pharmaceutical care, T2DM, and HRQoL. The search terms used included "pharmaceutical care" or "pharmacist" with "intervention" and "diabetes type 2" or "diabetes mellitus" and "quality of life." In addition, the bibliographies of all retrieved articles were handsearched to obtain additional articles not captured by the search strategy.

Studies included randomized controlled trials, clustered randomized controlled trials, and quasi-experimental studies with a single intervention group or those including a comparison group. The data extracted included year of publication, study design, study population, setting, duration of the trial, types and frequency of interventions, number of pharmacists involved, and economic, clinical, and patient-focused outcomes. The study setting could be a community pharmacy, outpatient clinic, or hospital.

The types of interventions included combinations of any of the following: diabetes education or information, medication review or counseling or adherence support, support of selfmanagement with respect to diet and lifestyle, self-monitoring of blood glucose, therapeutic recommendations or adjustment, and independent pharmacist medication management.

Types of clinical outcomes included fasting blood glucose, glycosylated hemoglobin (ie, $\mathrm{HbA}_{1 c}$ ), blood pressure, 
lipids (including total cholesterol, low-density lipoprotein cholesterol, high-density lipoprotein cholesterol, triglycerides), and body mass index. Patient-focused outcomes included HRQoL (both general and diabetes-specific), patient satisfaction, attitudes, and diabetes self-care activities. However, in assessing impact of pharmacists' diabetes services, two key outcomes were focused on: HRQoL and $\mathrm{HbA}_{1 \mathrm{c}}$.

Articles were selected by two independent reviewers and any disagreements were resolved.

\section{Quality assessment}

The quality of selected articles was examined using a validated checklist. ${ }^{32}$ The original Downs and Black instrument comprises 28 items that yield a maximum score of 32 ; several items that were not applicable to health service research trials were omitted, thus yielding a maximum possible score of 25 .
The checklist items address quality of reporting, internal and external validities, bias, confounding, and power. The score is expressed as a proportion, thus a score of one represents the highest quality and zero represents the lowest. A score of less than 0.5 is considered weak; $0.5-0.69$ fair, $0.7-0.79$ good, and $0.8-1.00$ very good..$^{32}$

\section{Results}

The results of the systematic review of all databases yielded 122 abstracts that addressed "pharmaceutical care" in T2DM (Figure 1). After the review of abstracts and handsearching of references, a total of 17 articles evaluating interventions delivered by a pharmacist either alone or a part of a team care arrangement for patients with T2DM, which included measurement of HRQoL, were suitable for inclusion in this review.

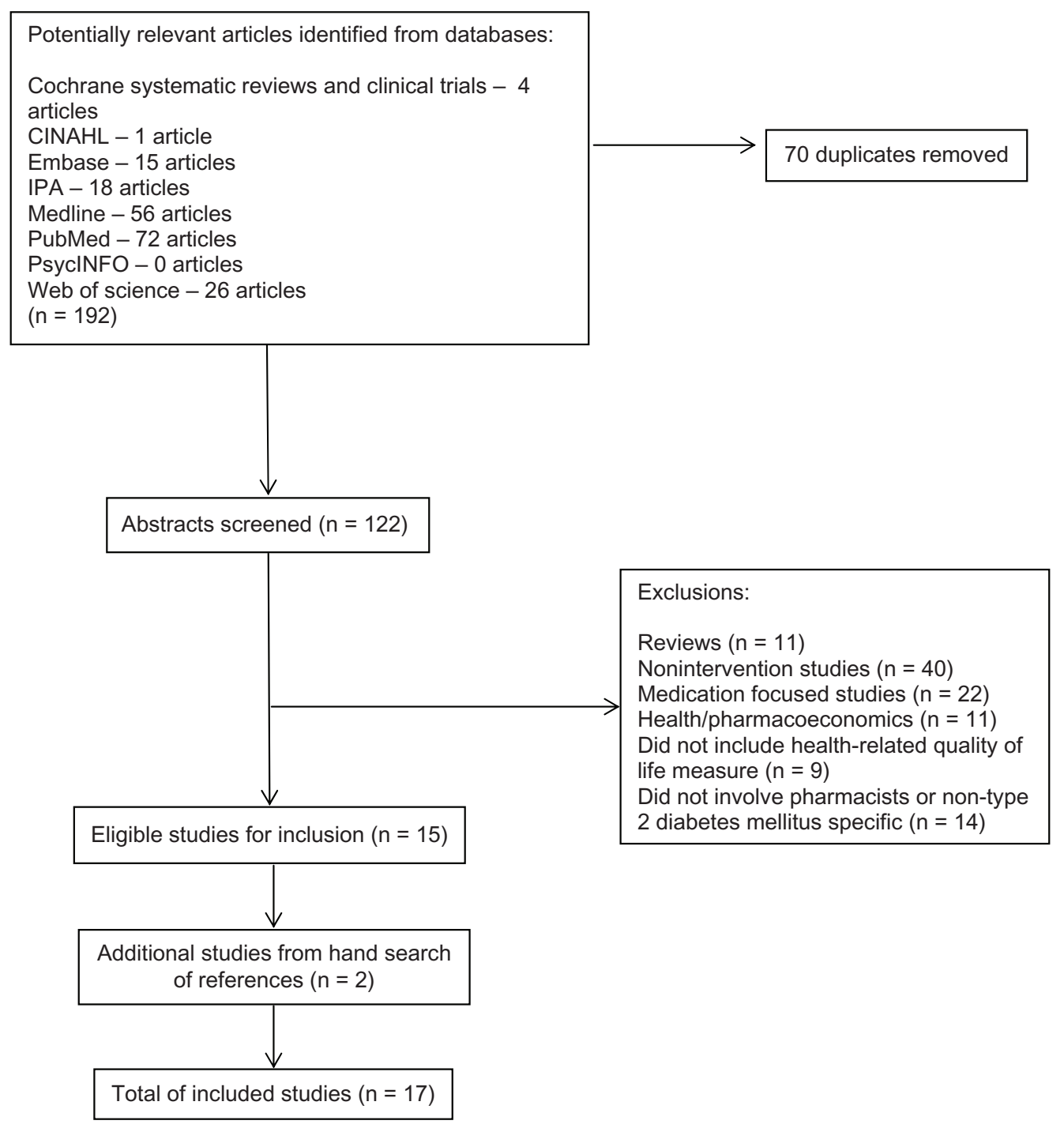

Figure I Literature search results. 
The studies included in this review span a time period of 26 years. Of the 17 included in this review, four were conducted in Australia, ${ }^{33-36}$ two in Brazil, ${ }^{37,38}$ one in Canada, ${ }^{39}$ two in India, ${ }^{40,41}$ two in the United Arab Emirates, ${ }^{42,43}$ one in the UK, ${ }^{44}$ and five in the USA ${ }^{45-49}$ (Table 1). Ten studies were conducted in community pharmacy settings ${ }^{33,35-39,42,44-46}$ and involved between one ${ }^{37,39}$ and $83^{36}$ community pharmacies in the provision of care, five were conducted in health centers or ambulatory clinics, ${ }^{34,40,47-49}$ and two in hospitals settings ${ }^{41,43}$ (Table 1). In eight studies, patients were randomized into intervention and control groups, ${ }^{34,39-41,43,44,47,49}$ two studies used a clustered randomized controlled design, ${ }^{35,36}$ one a parallel groups design, ${ }^{33}$ and four involved a single group repeated measures design (Table 1). ${ }^{37,42,45,48}$ The mean quality score for the 13 studies included in the quality rating was $0.63 \pm 0.11$ (range $0.40-0.76$ ), which is classified as only fair (Table 2).

\section{Pharmacist providers and interventions}

In eight studies, ${ }^{35,36,38,39,42,44-46}$ the pharmacists who provided the diabetes interventions were community pharmacists, and had received additional training in diabetes in four ${ }^{11,35,44,46}$ of the eight studies (Table 1). Two studies used credentialed diabetes educators ${ }^{39,45}$ and four specified clinical pharmacists. ${ }^{30,37,48,49}$ Overall, most studies lacked external validity and used small numbers of pharmacists in a limited number of settings. The exception to this were the studies by Krass et al which selected - either randomly or by quota sampling - between 28-83 pharmacies, which were representative Australian community pharmacies. ${ }^{35,36}$

Pharmacists' interventions spanned a range of activities and varied from a singular focus on medicine-related issues to a more comprehensive disease management approach to support people with T2DM. The duration of the intervention ranged from 3-12 months, and the frequency of contact with the pharmacists varied from weekly to monthly visits. All studies included some level of diabetes education/counseling mostly on an individual basis; only two studies involved prior group diabetes education sessions (Table 1)..$^{48,49}$ Medication review or counseling formed a part of the care package in several studies, ${ }^{33,35,36,38,39,41,43,44,47,49}$ while support of self-management with respect to diet and lifestyle $33,35,36,42$ and self-monitoring of blood glucose $\mathrm{e}^{33,35,36,39,43,46,47,49}$ were also mentioned by several (Table 1). Therapeutic recommendations to physicians featured in some studies, ${ }^{34,39,43,46,49}$ while independent therapeutic adjustment by the pharmacist occurred in only one trial (Table 1). ${ }^{47}$ Notably, no studies described a theoretical underpinning of the pharmacist intervention in the main outcomes paper.

\section{Impact of the pharmacist interventions on HRQoL}

Of the 17 studies that included a measure of impact on HRQoL, twelve used generic instruments: four used the SF-36, ${ }^{37,42-44}$ one used the SF-20, ${ }^{45}$ three used the SF-12, ${ }^{39,46,48}$ two used the EQ-5D, ${ }^{35,36}$ one used the Health Status Questionnaire (HSQ), ${ }^{47}$ and one used a nonspecified measure ${ }^{40}$ (Table 1). Only six studies used diabetes-specific HRQoL scales: four used the DQOL ${ }^{34,38,44,49}$ and two used the ADDQoL. ${ }^{33,41}$ One study used both a generic and diabetesspecific measure (Table 1). ${ }^{44}$

Overall, 13 of the 17 studies reported significant improvements in overall or subscale scores postintervention (Table 2). ${ }^{35-45,48,49}$ The size of the effect varied as did the detail in reporting of overall versus subscale scores. Because of the different scales used and the level of detail, it is difficult to compare between studies. Of the studies which used the SF-36 instrument, only two showed large improvements (according to the interpretation of SF-36 outcomes) ${ }^{28}$ in physical functioning and mental health scores, and these corresponded to large reductions in $\mathrm{HbA}_{1 \mathrm{c}} \cdot{ }^{37,43}$ One showed moderate effects ${ }^{39}$ and two showed small improvements in mental health. ${ }^{45,48}$ Several studies only showed impact in some HRQoL subscales and not others. For example, two studies reported significant improvements in mental health subscales with no significant change in physical functioning. ${ }^{39,48}$ It should be noted, however, that the mental health score at baseline in the intervention group in one of those studies was significantly lower than the baseline score of the control group and this may have contributed to the difference in mental health outcomes identified. ${ }^{39}$ Of the remaining four studies which did not report a significant improvement postintervention, two used a generic measure ${ }^{46,47}$ and two used diabetes-specific QoL measures. ${ }^{33,34}$ In several studies where there were baseline differences in HRQoL scores between intervention and control subjects, the appropriate statistical analysis to control for baseline differences was not conducted. ${ }^{43,44,49}$ Only four studies conducted a multivariate analysis to determine the impact of the intervention on HRQoL scores (Table 2). ${ }^{35,36,38,39}$

\section{Impact of the pharmacist interventions on $\mathrm{HbA}_{\mathrm{Ic}}$}

The key clinical outcome for 14 of the 17 studies was $\mathrm{HbA}_{1 \mathrm{c}} \cdot{ }^{33-37,39,41,43-49}$ In all but one study, ${ }^{34}$ there were 
Table I Characteristics of included studies

\begin{tabular}{|c|c|c|c|c|c|}
\hline Author & Setting (country) & Type of study & Patient population & $\begin{array}{l}\text { Number } \\
\text { of patients }\end{array}$ & $\begin{array}{l}\text { Lost to } \\
\text { follow-up }\end{array}$ \\
\hline \multicolumn{6}{|c|}{ Comparative studies } \\
\hline Al Mazroui & A 400-bed hospital & RCT & T2DM, taking OGLM, enrolled & 240 patients & I: 3 (2.5\%) \\
\hline \multirow[t]{2}{*}{ et $\mathrm{al}^{43}$} & (United Arab Emirates) & (PC versus UC) & in a military hospital & $\mathrm{I}: 120$ & C: $3(2.5 \%)$ \\
\hline & & & & C: 120 & \\
\hline
\end{tabular}

\begin{tabular}{|c|c|c|c|c|c|}
\hline Ali et $\mathrm{al}^{44}$ & $\begin{array}{l}\text { Two community } \\
\text { pharmacies (UK) }\end{array}$ & $\begin{array}{l}\mathrm{RCT} \\
\text { (PC versus UC) }\end{array}$ & $\begin{array}{l}\text { T2DM, age }>18 \text { years, taking } \\
\text { OGLM, not on insulin, } \\
\text { no significant comorbidity, } \\
\mathrm{HbA}_{\mathrm{Ic}} \geq 7 \%\end{array}$ & $\begin{array}{l}48 \text { patients } \\
\text { I: } 23 \\
\text { C: } 25\end{array}$ & $\begin{array}{l}\text { I: } 0(0.0 \%) \\
\text { C: } 2(8.0 \%)\end{array}$ \\
\hline Armour et $\mathrm{al}^{33}$ & $\begin{array}{l}\text { Community pharmacies } \\
\text { and diabetes clinics } \\
\text { I: } 6 \text { pharmacies, I clinic } \\
\text { C: } 6 \text { pharmacies, I clinic } \\
\text { (New South Wales, Australia) }\end{array}$ & $\begin{array}{l}\text { Controlled parallel } \\
\text { groups design }\end{array}$ & $\begin{array}{l}\text { T2DM, age } 18-85 \text { years, taking } \\
\text { more than } 3 \text { medications }\end{array}$ & $\begin{array}{l}239 \text { patients } \\
\text { I: } 128 \\
\text { C: III }\end{array}$ & $\begin{array}{l}\text { I: } 22(17.2 \%) \\
\text { C: } 29 \\
(26.1 \%)\end{array}$ \\
\hline Arun et $\mathrm{al}^{40}$ & $\begin{array}{l}3 \text { primary health centers } \\
\text { (India) }\end{array}$ & $\begin{array}{l}\text { Open-label parallel } \\
\text { study }\end{array}$ & $\begin{array}{l}\text { T2DM, age }>18 \text { years, } \\
\text { taking antidiabetic medication } \\
\text { for at least } 12 \text { months }\end{array}$ & $\begin{array}{l}\text { I54 patients } \\
\text { I: } 104 \\
\text { C: } 50\end{array}$ & $\mathrm{~N} / \mathrm{A}$ \\
\hline Correr et al ${ }^{38}$ & $\begin{array}{l}\text { Community pharmacies } \\
\text { and PCU } \\
\text { I: } 4 \text { pharmacies } \\
\text { C: I pharmacy and } \\
\text { I PCU (Brazil) }\end{array}$ & $\begin{array}{l}\text { Controlled } \\
\text { nonrandomized } \\
\text { trial }\end{array}$ & $\begin{array}{l}\text { T2DM, age }>30 \text { years, } \\
\text { taking OGLM or insulin }\end{array}$ & $\begin{array}{l}\text { I6I patients, } \\
96 \text { of whom } \\
\text { completed } \\
\text { the follow-up } \\
\text { I: } 50 \\
\text { C: } 46\end{array}$ & $65(40.4 \%)$ \\
\hline Clifford et $\mathrm{al}^{34}$ & $\begin{array}{l}\text { Diabetes outpatient clinic in } \\
\text { a hospital (Perth, Australia) }\end{array}$ & $\begin{array}{l}\mathrm{RCT} \\
\text { (PC versus UC) }\end{array}$ & $\begin{array}{l}\text { TIDM or T2DM, age }>18 \text { years, } \\
\text { high risk for developing diabetes } \\
\text { complication }\end{array}$ & $\begin{array}{l}73 \text { patients } \\
\text { I: } 48 \\
\text { C: } 25\end{array}$ & $\begin{array}{l}\text { I: } 0(0.0 \%) \\
\text { C: } 0(0.0 \%)\end{array}$ \\
\hline $\begin{array}{l}\text { Cranor and } \\
\text { Christensen }^{46}\end{array}$ & $\begin{array}{l}\text { I } 2 \text { community pharmacies } \\
\text { (North Carolina, USA) }\end{array}$ & $\begin{array}{l}\text { QE with a } \\
\text { comparison group } \\
\text { (PC versus UC) }\end{array}$ & Diabetes, no age restriction & $\begin{array}{l}85 \text { patients } \\
\text { I: } 38 \\
\text { C: } 47\end{array}$ & $\begin{array}{l}\text { I: } 0(0.0 \%) \\
\text { C: } 0(0.0 \%)\end{array}$ \\
\hline $\begin{array}{l}\text { Guirguis } \\
\text { et } \mathrm{al}^{39}\end{array}$ & $\begin{array}{l}\text { Community pharmacy } \\
\text { I: I } \\
\text { C: } 21\end{array}$ & $\mathrm{RCT}$ & $\begin{array}{l}\text { T2DM (for } \geq \mathrm{I} \text { year), } \\
\text { age }>\text { I } 8 \text { years, } \\
\text { noninstitutionalized }\end{array}$ & $\begin{array}{l}49 \text { patients } \\
\text { I: } 26 \\
\text { C: } 23\end{array}$ & $\begin{array}{l}\text { I: } 7(26.9 \%) \\
\text { C: } 6(26.1 \%)\end{array}$ \\
\hline
\end{tabular}

(Edmonton, Canada) 


\begin{tabular}{|c|c|c|c|c|c|}
\hline Interventionists & Pharmacist intervention & $\begin{array}{l}\text { Period } \\
\text { (months) }\end{array}$ & $\begin{array}{l}\text { Clinical } \\
\text { measures }\end{array}$ & Humanistic measures & $\begin{array}{l}\text { Economic } \\
\text { measures }\end{array}$ \\
\hline A research pharmacist & $\begin{array}{l}\text { I. Educate patient on medication and their } \\
\text { disease } \\
\text { 2. Advice on SMBG, physical exercise, } \\
\text { diet, medication adherence, and } \\
\text { smoking cessation } \\
\text { 3. Discussions with physician about } \\
\text { medication treatment } \\
\text { Frequency: every month }\end{array}$ & 12 & $\begin{array}{l}\mathrm{HbA}_{\mathrm{Ic}} \\
\text { FBG } \\
\mathrm{BMI} \\
\text { BP } \\
\text { Lipid panel }\end{array}$ & $\begin{array}{l}\text { HRQoL }(S F-36)^{60} \\
\text { Medication knowledge } \\
\text { (nonspecified instrument) } \\
\text { Self-reported medication } \\
\text { adherence }\end{array}$ & No \\
\hline $\begin{array}{l}3 \text { community } \\
\text { pharmacists who had } \\
\text { completed an 8-hour } \\
\text { training workshop }\end{array}$ & $\begin{array}{l}\text { I. Diabetes education } \\
\text { 2. Medicine use review } \\
\text { 3. Lifestyle modification counseling } \\
\text { 4. Referral to other health care professional } \\
\text { Frequency: every month for the first } \\
2 \text { months and then every } 3 \text { months } \\
\text { for the remainder }\end{array}$ & 12 & $\begin{array}{l}\mathrm{HbA}_{\text {Ic }} \\
\text { Blood glucose } \\
\text { BMI } \\
\text { BP } \\
\text { Lipid panel }\end{array}$ & $\begin{array}{l}\text { HRQoL }(\text { SF- } 36)^{27} \\
\text { Diabetes quality of life } \\
\text { (DQOL) }^{61} \\
\text { Diabetes knowledge (DKT) } \\
\text { Beliefs about medicines } \\
\left(\text { BMQ) }{ }^{62}\right. \\
\text { Satisfaction (SIMS) }{ }^{63}\end{array}$ & No \\
\hline $\begin{array}{l}\text { Pharmacist who had } \\
\text { completed a 2-day } \\
\text { training workshop }\end{array}$ & $\begin{array}{l}\text { I. Diabetes education } \\
\text { 2. Medication review and adherence support } \\
\text { 3. Support of SMBG } \\
\text { 4. Lifestyle advice } \\
\text { Frequency: approximately every month }\end{array}$ & 9 & $\mathrm{HbA}_{\mathrm{Ic}}$ & $\begin{array}{l}\text { Quality of life (ADDQoL) } \\
\text { Wellbeing (WB-QI2) } \\
\text { Medication adherence } \\
\text { (Brief Medication } \\
\text { Questionnaire) }\end{array}$ & $Y_{e s}^{51}$ \\
\hline Pharmacists & $\begin{array}{l}\text { Counseling about diabetes and health care } \\
\text { Frequency: every month }\end{array}$ & 6 & $\begin{array}{l}\text { FBG } \\
\text { Body weight } \\
\text { BMI } \\
\text { BP }\end{array}$ & $\begin{array}{l}\text { HRQoL (nonspecified } \\
\text { instrument) }\end{array}$ & No \\
\hline $\begin{array}{l}\text { Community } \\
\text { pharmacists who had } \\
\text { received a training in } \\
\text { pharmacotherapeutic } \\
\text { follow-up using the } \\
\text { Dader method }\end{array}$ & $\begin{array}{l}\text { Pharmacotherapeutic follow-up } \\
\text { (Dader method): } 6 \\
\text { I. Patient recruitment } \\
\text { 2. Clinical interview } \\
\text { 3. Detection of DTPs } \\
\text { 4. Plan of pharmaceutical care } \\
\text { and intervention } \\
\text { 5. Evaluation of results }\end{array}$ & 12 & N/A & $\begin{array}{l}\text { HRQoL (DQOL-Brazil, } \\
\text { validated in Portuguese) } \\
\text { Satisfaction with pharmacy } \\
\text { services }\end{array}$ & No \\
\hline A clinical pharmacist & $\begin{array}{l}\text { Review and monitor all aspects of the } \\
\text { patients' drug therapy in collaboration } \\
\text { with other health care professionals } \\
\text { Frequency: 6-week intervals }\end{array}$ & 6 & $\mathrm{HbA}_{\mathrm{Ic}}$ & $\begin{array}{l}\text { QoL (DQOL) } \\
\text { Satisfaction (nonspecified } \\
\text { instrument) }\end{array}$ & No \\
\hline $\begin{array}{l}\text { Community } \\
\text { pharmacists who } \\
\text { had completed } \\
\text { a certificate training } \\
\text { program in diabetes }\end{array}$ & $\begin{array}{l}\text { I. Diabetes education } \\
\text { 2. Training in SMBG } \\
\text { 3. Monitoring and follow up } \\
\text { 4. Therapeutic recommendations } \\
\text { and referral } \\
\text { Frequency: every month }\end{array}$ & $7-9$ & $\begin{array}{l}\mathrm{HbA}_{\text {Ic }} \\
\text { Lipid panel }\end{array}$ & $\begin{array}{l}\text { HRQoL (SF-I } 2)^{68} \\
\text { Satisfaction with } \\
\text { pharmacy services }^{69}\end{array}$ & Yes \\
\hline 2 CDE pharmacists & $\begin{array}{l}\text { I. Educate on SMBG } \\
\text { 2. Diabetes education } \\
\text { 3. Lifestyle, nutrition, exercise, } \\
\text { and medication use } \\
\text { 4. Monitoring and therapeutic } \\
\text { recommendation to physicians } \\
\text { Frequency: } 2 \text { times in the first month } \\
\text { and then approximately every month } \\
\text { for the remainder }\end{array}$ & 6 & $\mathrm{HbA}_{\mathrm{Ic}}$ & $\begin{array}{l}\text { HRQoL }(\mathrm{SF}-\mathrm{I} 2)^{68} \\
\text { Attitudes to diabetes (DAS) } \\
\text { Self care activities } \\
\text { (SDSCA) } \\
\text { Satisfaction with } \\
\text { pharmacy services }^{70}\end{array}$ & No \\
\hline
\end{tabular}


Table I (Continued)

\begin{tabular}{llllll}
\hline Author & Setting (country) & Type of study & Patient population & $\begin{array}{l}\text { Number } \\
\text { of patients }\end{array}$ & $\begin{array}{l}\text { Lost to } \\
\text { follow-up }\end{array}$ \\
\hline Jaber et al ${ }^{47}$ & Medicine outpatient clinic & RCT & T2DM & 45 patients & I: $6(26.1 \%)$ \\
& (USA) & (PC versus UC) & & I: 23 & C: $0(0.0 \%)$ \\
& & & C: 22 &
\end{tabular}

\begin{tabular}{|c|c|c|c|c|c|}
\hline Krass et a $\left.\right|^{35}$ & $\begin{array}{l}56 \text { community pharmacies } \\
\text { I: } 28 \\
\text { C: } 28 \\
\text { (Australia) }\end{array}$ & $\begin{array}{l}\text { Clustered } \\
\text { RCT } \\
\text { (DMAS versus UC) }\end{array}$ & $\begin{array}{l}\text { T2DM and (a) taking } \geq I \text { OGLM } \\
\text { or insulin ( } \mathrm{HbA}_{\mathrm{Ic}} \geq 7.5 \% \text { ) or } \\
\text { (b) taking } \geq I \text { OGLM or insulin } \\
\text { and } \geq I \text { antihypertensive, angina, } \\
\text { or lipid-lowering drugs }\end{array}$ & $\begin{array}{l}335 \text { patients } \\
\text { I: } 176 \\
\text { C: } 159\end{array}$ & $\begin{array}{l}\text { I: } 27(15.3 \%) \\
\text { C: } 19 \\
(11.9 \%)\end{array}$ \\
\hline Krass et $\mathrm{a}^{36}$ & $\begin{array}{l}83 \text { community pharmacies } \\
\text { I: } 42 \\
\text { C: } 4 \mathrm{I} \\
\text { (Australia) }\end{array}$ & $\begin{array}{l}\text { Clustered } \\
\text { RCT } \\
6 \text { months pharmacist } \\
\text { DMAS versus } \\
\text { I } 2 \text { months DMAS }\end{array}$ & T2DM with $\mathrm{HbA}_{\mathrm{Ic}}>7.0 \%$ & $\begin{array}{l}524 \text { patients } \\
\text { I: } 260 \\
\text { C: } 264\end{array}$ & $\begin{array}{l}\text { I: } 57(21.9 \%) \\
\text { C: } 80 \\
(30.3 \%)\end{array}$ \\
\hline Scott et $\mathrm{al}^{49}$ & $\begin{array}{l}\text { Community health center } \\
\text { (lowa, USA) }\end{array}$ & $\begin{array}{l}\mathrm{RCT} \\
\text { (PC versus } \mathrm{UC})\end{array}$ & T2DM, age $>18$ years & $\begin{array}{l}\text { I } 49 \text { patients } \\
\text { I: } 76 \\
\text { C: } 73\end{array}$ & $\begin{array}{l}\text { I: } 12(15.8 \%) \\
\text { C: } 6(8.2 \%)\end{array}$ \\
\hline Sriram et $\mathrm{al}^{41}$ & $\begin{array}{l}\text { Hospital } \\
\text { (South India) }\end{array}$ & $\begin{array}{l}\mathrm{RCT} \\
\text { (PC versus } \mathrm{UC})\end{array}$ & T2DM, age $>18$ years & $\begin{array}{l}\text { I } 20 \text { patients } \\
\text { I: } 60 \\
\text { C: } 60\end{array}$ & $\mathrm{~N} / \mathrm{A}$ \\
\hline \multicolumn{6}{|c|}{ Single group studies } \\
\hline $\begin{array}{l}\text { Abduelkarem } \\
\text { and Sackville }\end{array}$ & $\begin{array}{l}3 \text { community pharmacies } \\
\text { (United Arab Emirates) }\end{array}$ & $\begin{array}{l}\mathrm{QE} \\
\text { (Single group } \\
\text { repeated measures) }\end{array}$ & T2DM, age up to 85 years & 59 patients & $\mathrm{N} / \mathrm{A}$ \\
\hline Baran et $\mathrm{a}^{45}$ & $\begin{array}{l}10 \text { pharmacists in different } \\
\text { community settings (USA) }\end{array}$ & $\begin{array}{l}\mathrm{QE} \\
\text { (Single group } \\
\text { repeated measures) }\end{array}$ & $\begin{array}{l}\text { TIDM or T2DM, } \\
\text { age }>55 \text { years }\end{array}$ & 88 patients & $\mathrm{N} / \mathrm{A}$ \\
\hline $\begin{array}{l}\text { Balisa-Rocha } \\
\text { et al }{ }^{37}\end{array}$ & $\begin{array}{l}\text { A community pharmacy } \\
\text { (Brazil) }\end{array}$ & $\begin{array}{l}\mathrm{QE} \\
\text { (Single group } \\
\text { repeated measures) }\end{array}$ & $\begin{array}{l}\text { T2DM, age } 60-75 \text { years, } \\
\text { taking antidiabetic medication }\end{array}$ & 50 patients & $16(32.0 \%)$ \\
\hline Johnson et $\mathrm{al}^{48}$ & $\begin{array}{l}\text { Ambulatory care facility } \\
\text { (University of Kentucky, } \\
\text { USA) }\end{array}$ & $\begin{array}{l}\text { Retrospective audit } \\
\text { of clinic data }\end{array}$ & Adult with diabetes & I0I patients & N/A \\
\hline
\end{tabular}

Abbreviations: ADDQoL, Audit of Diabetes-Dependent Quality of Life; BMI, body mass index; BMQ, Beliefs about Medicines Questionnaire; BP, blood pressure; C, controlled; CDE, credentialed diabetes educator; DAS, Diabetes Attitude Scale; DKT, Diabetes Knowledge Test; DMAS, Diabetes Medication Assistance Service; DQOL, Diabetes Quality of Life questionnaire; DRPs, drug-related problems; DTSQ, Diabetes Treatment Satisfaction Questionnaire; EQ-5D, EuroQoL-5 Dimension; FBG, fasting blood glucose; GP, general practitioner; HbA , glycosylated hemoglobin; HRQoL, health-related quality of life; HSQ, Health Status Questionnaire; I, intervention; OGLM, oral glucose-lowering medication; PC, pharmaceutical care; PCU, primary care unit; QE, quasi experimental; RCT, randomized controlled trial; SDSCA, Summary of Diabetes Self-Care Activities; SF, Short Form; SIMS, Satisfaction with Information about Medicines Scale; SMBG, self-monitoring of blood glucose; TIDM, type I diabetes mellitus; T2DM, type 2 diabetes mellitus; UC, usual care; WB-QI2, Well-Being Questionnaire-12. 


\begin{tabular}{|c|c|c|c|c|c|}
\hline Interventionists & Pharmacist intervention & $\begin{array}{l}\text { Period } \\
\text { (months) }\end{array}$ & $\begin{array}{l}\text { Clinical } \\
\text { measures }\end{array}$ & Humanistic measures & $\begin{array}{l}\text { Economic } \\
\text { measures }\end{array}$ \\
\hline A pharmacist & $\begin{array}{l}\text { I. Pharmacotherapeutic evaluation } \\
\text { and dosage adjustment } \\
\text { 2. Diabetes education } \\
\text { 3. Medication counseling } \\
\text { 4. Instruction on diet and exercise } \\
\text { 5. Training for SMBG } \\
\text { Frequency: every week until glycemic } \\
\text { control achieved, then every } 2-4 \text { weeks }\end{array}$ & 4 & $\begin{array}{l}\text { FBG } \\
\mathrm{HbA} \\
\text { BP } \\
\text { Lipid panel }\end{array}$ & $\begin{array}{l}\text { HRQoL (HSQ } \\
\text { version } 2.0)^{71}\end{array}$ & No \\
\hline $\begin{array}{l}\text { Community } \\
\text { pharmacists who } \\
\text { had completed } \\
\text { a 2-day workshop }\end{array}$ & $\begin{array}{l}\text { I. Review of SMBG } \\
\text { 2. Educate on disease, medication, and lifestyle } \\
\text { 3. Self-management support } \\
\text { 4. Detection of DRPs } \\
\text { 5. Referrals to the GP when appropriate } \\
\text { Frequency: } 5 \text { times over } 6 \text { months }\end{array}$ & 6 & $\begin{array}{l}\mathrm{HbA}_{\mathrm{Ic}} \\
\mathrm{BP} \\
\text { Lipid panel } \\
\text { BMI }\end{array}$ & HRQoL (EQ-5D) ${ }^{72}$ & $Y_{e s}{ }^{52}$ \\
\hline $\begin{array}{l}\text { Credentialed } \\
\text { pharmacist to } \\
\text { deliver DMAS }\end{array}$ & $\begin{array}{l}\text { I. Review of SMBG } \\
\text { 2. Educate on disease, medication, } \\
\text { and lifestyle } \\
\text { 3. Self-management support } \\
\text { 4. Detection of DRPs } \\
\text { 5. Referrals to the GP when appropriate } \\
\text { Frequency: } 4 \text { times over } 6 \text { months; } \\
\text { and } 6 \text { times over } 12 \text { months }\end{array}$ & 6 versus 12 & $\begin{array}{l}\mathrm{HbA}_{\mathrm{lc}} \\
\mathrm{BP} \\
\text { Lipid panel } \\
\text { BMl }\end{array}$ & HRQoL (EQ-5D) $)^{72}$ & No \\
\hline $\begin{array}{l}\text { A clinical pharmacist } \\
\text { who had completed } \\
\text { a I-year PC residency } \\
\text { program }\end{array}$ & $\begin{array}{l}\text { 2-hour group education; followed by } \\
\text { I. Support for SMBG } \\
\text { 2. Medication review } \\
\text { 3. Therapeutic recommendations } \\
\text { Frequency: every } 2 \text { weeks for } 3 \text { months } \\
\text { then every } 3 \text { months }\end{array}$ & 12 & $\begin{array}{l}\mathrm{HbA}_{\mathrm{Ic}} \\
\mathrm{BP} \\
\text { Lipid panel } \\
\text { BMl }\end{array}$ & HRQoL (DQOL) ${ }^{24}$ & No \\
\hline A clinical pharmacist & $\begin{array}{l}\text { I. Medication counseling } \\
\text { 2. Instruction on dietary regulation, } \\
\text { exercise, and other lifestyle modification } \\
\text { Frequency: N/A }\end{array}$ & 8 & $\begin{array}{l}\text { FBG } \\
\mathrm{HbA}_{\text {Ic }} \\
\mathrm{BMI}\end{array}$ & $\begin{array}{l}\text { HRQoL (ADDQoL) })^{25} \\
\text { Satisfaction (DTSQ) }{ }^{73}\end{array}$ & No \\
\hline $\begin{array}{l}\text { Community } \\
\text { pharmacists }\end{array}$ & $\begin{array}{l}\text { Reinforcement of diabetes self-management } \\
\text { information } \\
\text { Frequency: every week }\end{array}$ & $\begin{array}{l}3 \text { (I) and } 24 \\
\text { (follow-up) }\end{array}$ & BMI & $\begin{array}{l}\text { HRQoL }(\mathrm{SF}-36)^{27} \\
\text { Self-care activity }(\mathrm{SDSCA})^{74} \\
\text { Perception of the service }{ }^{75}\end{array}$ & No \\
\hline $\begin{array}{l}\text { Pharmacists qualified } \\
\text { as } C D E\end{array}$ & $\begin{array}{l}\text { I. Diabetes-specific counseling } \\
\text { 2. Educational materials } \\
\text { 3. Monitoring therapy } \\
\text { Frequency: at least every } 2 \text { months }\end{array}$ & 6 & $\begin{array}{l}\mathrm{HbA}_{\mathrm{lc}} \\
\text { Random blood } \\
\text { glucose } \\
\text { BP } \\
\text { Total cholesterol }\end{array}$ & HRQoL (SF-20) $)^{76}$ & No \\
\hline A clinical pharmacist & $\begin{array}{l}\text { I. Medication therapy management } \\
\text { 2. Educational interventions } \\
\text { Frequency: every month (approximately } \\
40-60 \text { minutes each session) }\end{array}$ & 10 & $\begin{array}{l}\mathrm{HbA}_{\mathrm{Ic}} \\
\text { Capillary blood } \\
\text { glucose } \\
\text { BP } \\
\text { BMI }\end{array}$ & HRQoL (SF-36) $)^{27}$ & No \\
\hline $3 \mathrm{CDE}$ pharmacists & $\begin{array}{l}\text { A 6-hour comprehensive group education } \\
\text { for diabetes self-management followed by } \\
\text { medication therapy management service } \\
\text { during maintenance visits } \\
\text { Frequency: every } 3 \text { months for } \\
\text { maintenance visits }\end{array}$ & 12 & $\begin{array}{l}\mathrm{HbA}_{\mathrm{Ic}} \\
\mathrm{BP} \\
\text { Lipid panel } \\
\text { BMI } \\
\text { Aspirin use and } \\
\text { vaccinations }\end{array}$ & $\begin{array}{l}\text { HRQoL (SF-I } 2)^{68} \\
\text { Satisfaction (Diabetes } \\
\text { History Form) }\end{array}$ & No \\
\hline
\end{tabular}




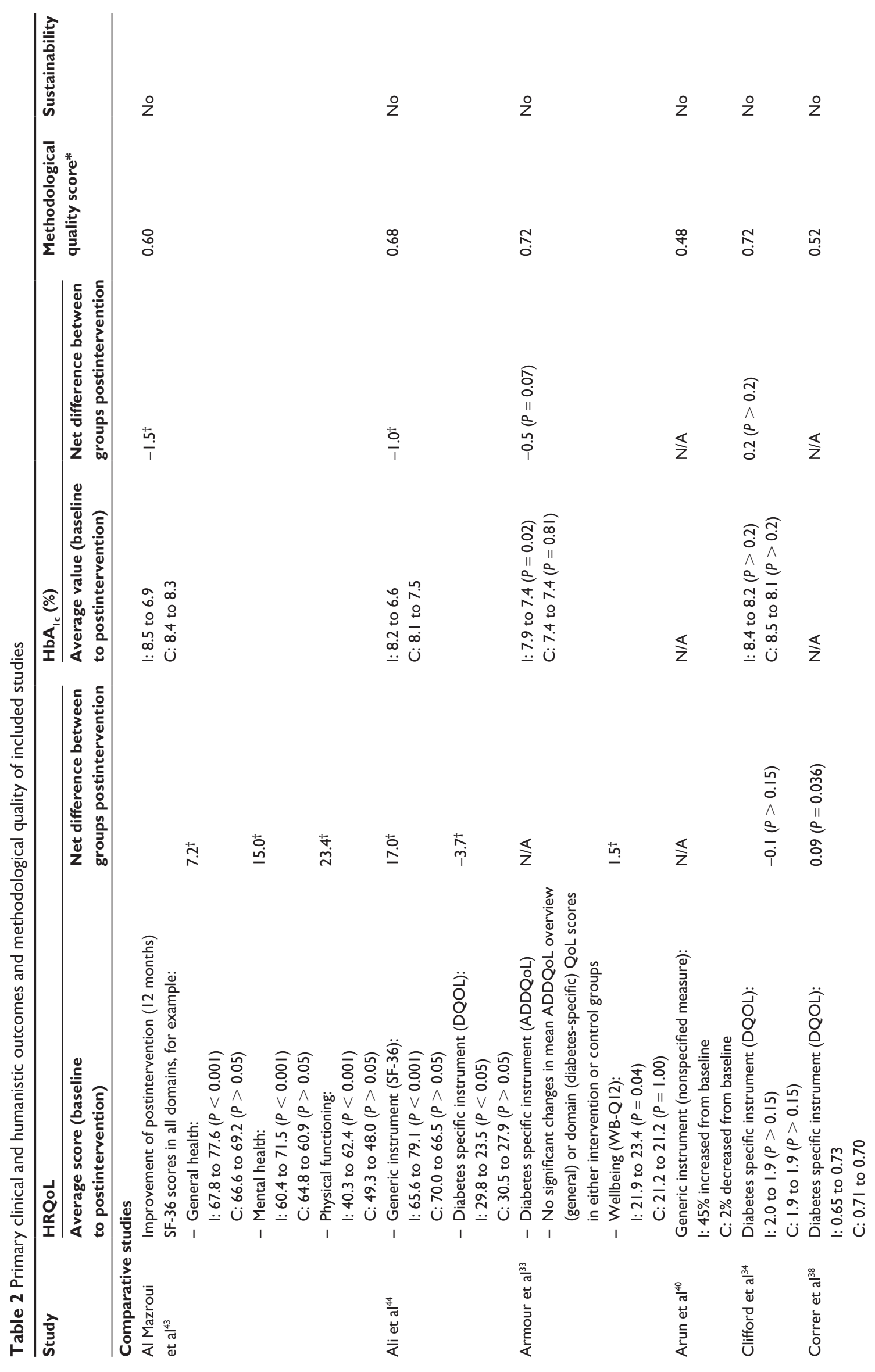




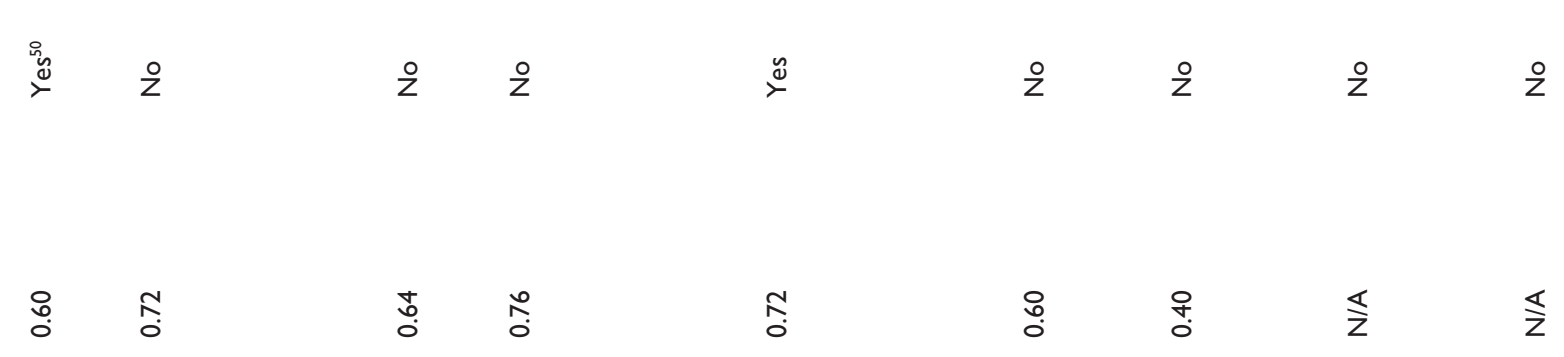

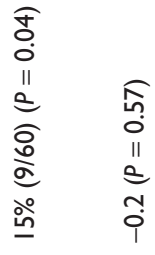

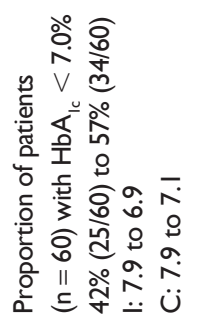

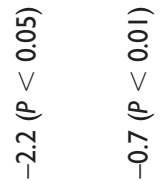

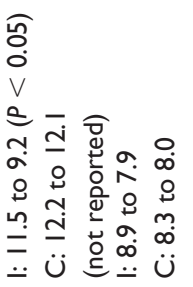

0
0
0
0
11
0
0
0

$\stackrel{+}{i}$

훟

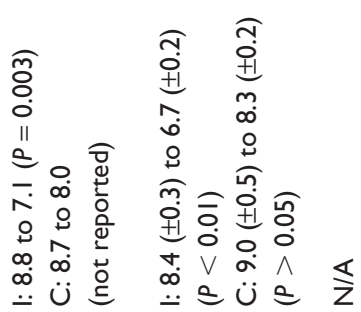

ํㅜㄴ

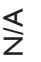

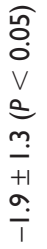

$\widehat{s}$

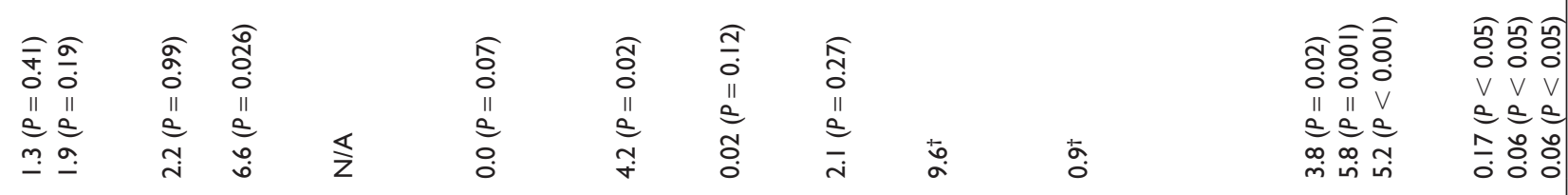

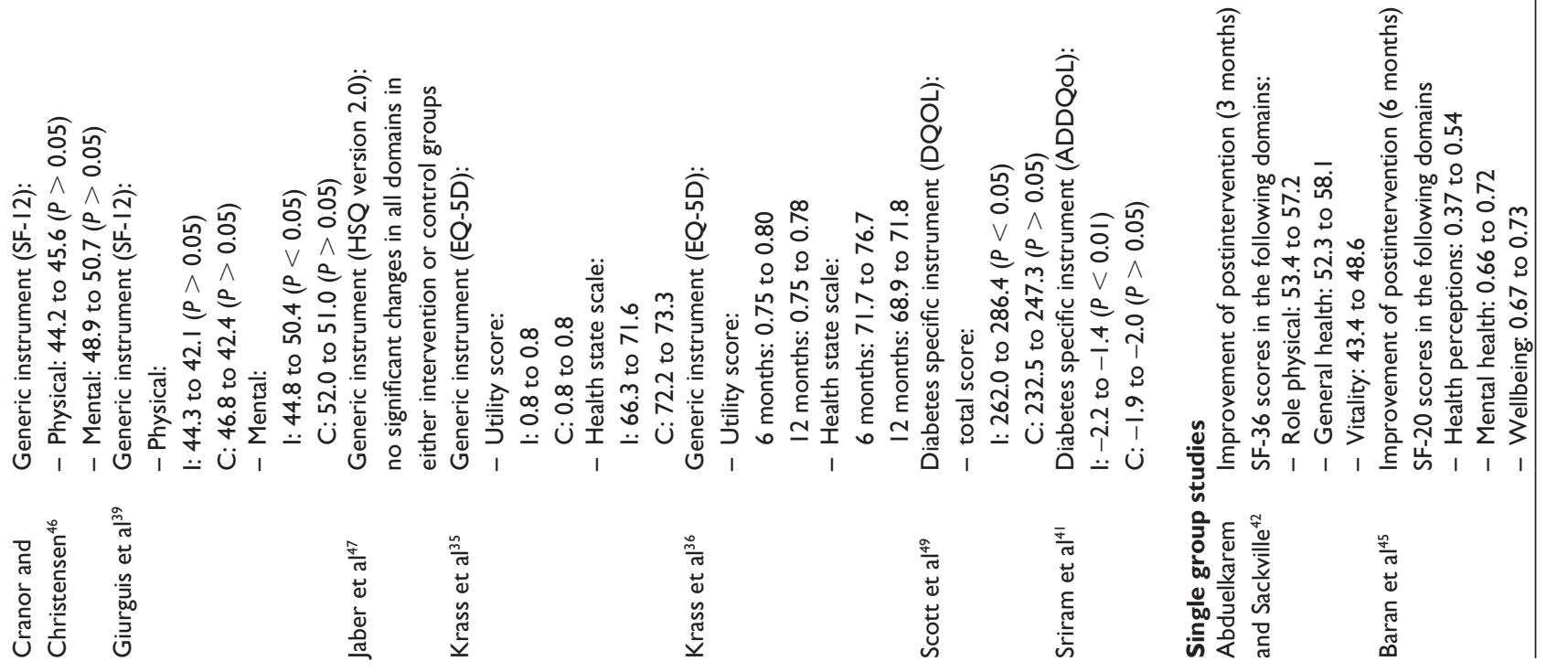




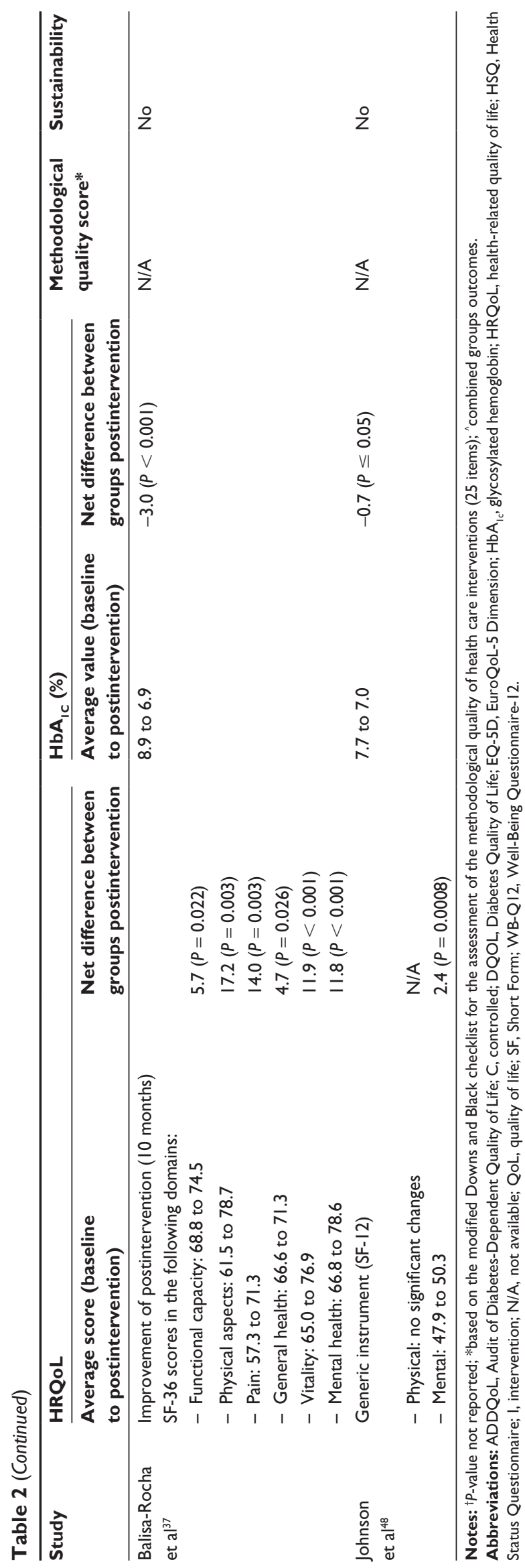

reductions in mean $\mathrm{HbA}_{1 \mathrm{c}}$ postintervention in the intervention group; however, the effect sizes ranged from $0.5 \%-3 \%$ with a median reduction of $1 \%$ (interquartile range $0.6-1.7$ ) (Table 2). The data suggests that the higher the $\mathrm{HbA}_{1 \mathrm{c}}$ was on entry into the program, the greater the reduction in $\mathrm{HbA}_{1 \mathrm{c}}$. Only one study reported the change in proportion of subjects who reached a target $\mathrm{HbA}_{1 \mathrm{c}}$ of $\leq 7 \% .{ }^{46}$

In the studies with control or comparison groups, the results mostly favored the intervention group; however, in one study, similar reductions in $\mathrm{HbA}_{1 \mathrm{c}}$ were seen in both the intervention and control groups (Table 2).$^{39}$ As with the analyses of HRQoL scores, only a few studies conducted multivariate analyses. Moreover, only two studies reported on the sustainability of glycemic control beyond the period of the intervention (Table 2). Krass et al followed Australian T2DM patients for up to 12 months following the Diabetes Medication Assistance Service intervention and found that improvements in $\mathrm{HbA}_{1 \mathrm{c}}$ had been sustained. ${ }^{11,36,50}$ In the USA, Cranor et al followed a cohort of T2DM patients who received ongoing pharmaceutical care services for up to 5 years and found that they also maintained improvements in $\mathrm{HbA}_{\mathrm{lc}}$ over time. ${ }^{50}$

\section{Other outcomes}

With respect to other clinical outcomes, positive impacts with statistically significant reductions were reported in six of ten studies that measured body mass index, ${ }^{40-44,47}$ in six of seven studies that measured fasting or random blood glucose, ${ }^{37,40,41,43,44,47}$ in six of ten studies that measured systolic blood pressure,,$^{37,40,43-45,49}$ and in four of ten studies that measured diastolic pressure..$^{37,43-45}$ Of the nine studies that measured lipids, five reported reduction in total cholesterol, ${ }^{11,43,45,48,49}$ three in low-density lipoprotein cholesterol ${ }^{43,48,49}$ and high-density lipoprotein cholesterol, ${ }^{43,44,48}$ and four in triglycerides. ${ }^{11,36,43,48}$

Positive impacts on other humanistic outcomes included improvements in knowledge, ${ }^{43,44}$ wellbeing, medication adherence, ${ }^{33,43}$ and service satisfaction. ${ }^{34,41,46,48}$

Only three studies have conducted an economic analysis. Cranor and Christensen conducted a cost analysis, which showed a reduction in nondiabetes-related costs following the pharmaceutical care intervention. ${ }^{46}$ Taylor et al conducted an incremental cost-effectiveness analysis on the intervention implemented by Armour et al..$^{33,51}$ The results showed that to obtain the $0.43 \%$ ( $95 \%$ confidence interval (CI) $0.34-0.52$ ) reduction in $\mathrm{HbA}_{1 \mathrm{c}}$ achieved by the pharmacist diabetes intervention, the cost to the health care sector was AUD383 (95\% CI AUD46.16-717.46) or USD388 
(95\% CI USD46.72-726.21) per patient per 9 months. An incremental cost-effectiveness and utility analysis of the Diabetes Medication Assistance Service ${ }^{35}$ was conducted by Krass et al. ${ }^{52}$ The cost effectiveness was found to compare favorably with other accepted uses of health care resources funded by the Australian government. The cost per life year saved was estimated to be from AUD17,752 (USD17,969) to AUD24,029 (USD24,322) and the cost per quality-adjusted life year from AUD22,486 (USD22,760) to AUD30,582 (USD30,955), depending on the scenario used. ${ }^{52}$

\section{Discussion}

The results of this review provide some preliminary evidence that pharmaceutical care and pharmacist disease management diabetes services can have a positive impact on HRQoL, with the evidence suggesting a greater effect on mental rather than physical health. It was also notable that the largest impacts on QoL were seen in studies in which only a small number of pharmacists were delivering the intervention. However, what is striking is that over the two decades of research and more than 100 published studies that have investigated the impact of pharmacists' services in T2DM, only 17 were found to have included HRQoL as an outcome measure. This is consistent with findings of previous systematic reviews of care management interventions which showed that HRQoL was not measured in most trials..$^{53,54}$ It is therefore not meaningful to compare the impact of diabetes interventions on HRQoL delivered by pharmacists with those of other health care professionals. It is also clear that the lack of rigorously designed trials using the same general and diabetes-specific HRQoL instruments make it impossible to conduct a metaanalysis. Thus, any firm conclusions about the extent to which pharmacist interventions impact on HRQoL in T2DM could not be drawn.

This contrasts with the information on the impact of pharmaceutical care and pharmacist disease management diabetes services on $\mathrm{HbA}_{1 \mathrm{c}}$ where it has been possible to pool outcomes from a number of randomized controlled trials and show that reductions that were achieved were both statistically significant and clinically important. The median reduction in $\mathrm{HbA}_{1 \mathrm{c}}$ of $1 \%$, observed in the studies included in this review, is consistent with weighted meta-analytic estimated differences reported by Machado et $\mathrm{al}^{13}$ as well the findings of a Canadian health technology assessment of specialized multidisciplinary community care for T2DM provided by at least a pharmacist and a primary care physician for the management of T2DM compared to usual care. ${ }^{55}$ Moreover, as has previously been reported, the higher the $\mathrm{HbA}_{1 \mathrm{c}}$ at baseline, the greater the mean reduction observed postintervention. ${ }^{56}$ Based on evidence from the UK Prospective Diabetes Study, the improvement in diabetes control achieved by the pharmacists' interventions are likely to translate into future cost savings to the health care system by delaying and reducing diabetes-related complications. ${ }^{5}$

Evidence for a relationship between glycemic control and HRQoL is currently inconclusive as some studies have shown the benefit of glycemic control on HRQoL, ${ }^{57}$ while others have reported contrary findings. ${ }^{58}$ This uncertainty was also observed in the studies included in this review as improvements in HRQoL were identified both in studies with improved or unchanged $\mathrm{HbA}_{1 \mathrm{c}}$ postintervention.

The variation in impacts on both clinical and humanistic outcomes may also be attributed to different intensities and types of intervention. Most pharmacists' interventions comprised a mix of some diabetes education with ongoing monitoring and support of self-management as well as identification and addressing of drug-related problems, with varying levels of focus on each of these elements. Importantly, in most studies, the pharmacist met with patients on a regular basis over a period of 6 months to 1 year. This was critical to the success of the program as intensive support to improve patients' self-management skills has previously been shown to improve glycemic control, patient satisfaction, and QoL. ${ }^{17,59}$ In terms of blood pressure and lipid control, it may be the case that studies that included a focus on medication review and referral for therapy intensification to achieve recommended therapeutic targets were more successful in achieving these outcomes.

Since both credentialed diabetes educators and pharmacists, who received only 1-2 days of additional training, were able to deliver diabetes care that translated into improvements in clinical and humanistic outcomes, the optimum training requirements to upskill pharmacists require further clarification. Moreover, given the complexity of the diabetes interventions delivered in most studies, it is impossible to pinpoint how and to what extent each element contributed most to the observed outcomes. As a corollary, it is also difficult to unravel which changes in patient or health care professional behaviors most contributed to the improvements seen in clinical and humanistic outcomes, eg, changes in patient behaviors such as better adherence to lifestyle recommendations, better adherence to medication, and more regular self-monitoring of blood glucose, or changes to therapy recommended or initiated by the pharmacist.

The lack of economic evaluation of pharmacists' diabetes interventions is also noteworthy. Even if pharmacists' 
diabetes interventions are shown to be clinically effective and able to improve HRQoL, in light of resources restraints in the health care system, without well-designed cost-utility studies demonstrating cost-effectiveness, it will be difficult to persuade governments or third-party payers to fund, subsidize, or support pharmacist diabetes interventions. Such health policy decisions in turn will influence the feasibility of widespread implementation of such services into clinical practice in community or hospital settings.

\section{Implications for patient management}

Chronic disease management of T2DM requires a mix of strategies that deliver optimum pharmacotherapy, patient education, and self-management support to the patient over the course of their disease. To achieve treatment targets required to prevent complications and maintain a good QoL for the patient, a team approach is needed, and pharmacists by virtue of their accessibility, medication expertise, good rapport, and frequent contact with patients should become active members of the diabetes care team.

\section{Conclusion}

The literature suggests that pharmaceutical care delivered by pharmacists in any clinical setting will make a positive contribution to clinical outcomes for patients with T2DM, especially glycemic control. Evidence for the impact of pharmaceutical care on HRQoL, however, is still limited and inconclusive. Future studies should use robust research designs to bolster the evidence for the impact of pharmaceutical care and pharmacist disease management diabetes services on HRQoL using both generic and disease-specific validated measures that are easy to administer and have proven sensitivity to change. Moreover, the sustainability of any improvements beyond the intervention period also needs to be determined. Further research is also needed to compare the impact of different intensities of intervention and follow-up as well as the individual components of interventions to identify the most feasible and effective approaches. All future studies should adopt the ECHO model which includes clinical, humanistic, and economic evaluation of interventions to usefully inform policy makers in decisions on whether to support the widespread implementation of pharmaceutical care as an evidence-based strategy in the management of T2DM.

\section{Disclosure}

The authors report no conflicts of interest in this work.

\section{References}

1. Shaw JE, Sicree RA, Zimmet PZ. Global estimates of the prevalence of diabetes for 2010 and 2030. Diabetes Res Clin Pract. 2010; 87(1):4-14.

2. World Health Assembly. Global strategy on diet, physical activity and health. In: Fifty-seventh World Health Assembly, Geneva, May 17-22, 2004. Resolutions and Decisions, Annexes. Geneva: World Health Organization; 2004.

3. Diabetes: fact sheet $N^{\circ} 312$ [webpage on the Internet]. Geneva: World Health Organization; 2012. Available from: http:/www.who.int/ mediacentre/factsheets/fs312/en/index.html. Accessed November 21, 2012.

4. UK Prospective Diabetes Study Group. Intensive blood-glucose control with sulphonylureas or insulin compared with conventional treatment and risk of complications in patients with type 2 diabetes (UKPDS33). Lancet. 1998;352(9131):837-853.

5. Stratton IM, Adler AI, Neil HA, et al. Association of glycaemia with macrovascular and microvascular complications of type 2 diabetes (UKPDS 35): prospective observational study. BMJ. 2000; 321(7258):405-412.

6. UK Prospective Diabetes Study Group. Tight blood pressure control and risk of macrovascular and microvascular complications in type 2 diabetes: UKPDS 38. BMJ. 1998;317(7160):703-713.

7. Proudfoot J, Infante F, Holton C, et al. Organisational capacity and chronic disease care: an Australian general practice perspective. Aust Fam Physician. 2007;36(4):286-288.

8. Harris M. Challenges in diabetes management. Aust Fam Physician. 2008;37(9):716-720.

9. Eliasson B, Cederholm J, Nilsson P, Gudbjornsdottir S. The gap between guidelines and reality: type 2 diabetes in a National Diabetes Register 1996-2003. Diabet Med. 2005;22(10):1420-1426.

10. Janus ED, Tideman PA, Dunbar JA, et al. Dyslipidaemia in rural Australia: prevalence, awareness, and adherence to treatment guidelines in the Greater Green Triangle Risk Factor Study. Med J Aust. 2010;192(3):127-132.

11. Krass I, Hebing R, Mitchell B, et al. Diabetes management in an Australian primary care population. J Clin Pharm Ther. 2011;36(6): 664-672.

12. Hepler CD, Strand LM. Opportunities and responsibilities in pharmaceutical care. Am J Hosp Pharm. 1990;47(3):533-543.

13. Machado M, Bajcar J, Guzzo GC, Einarson TR. Sensitivity of patient outcomes to pharmacist interventions. Part I: systematic review and meta-analysis in diabetes management. Ann Pharmacother. 2007;41(10):1569-1582.

14. Wubben DP, Vivian EM. Effects of pharmacist outpatient interventions on adults with diabetes mellitus: a systematic review. Pharmacotherapy. 2008;28(4):421-436.

15. Evans CD, Watson E, Eurich DT, et al. Diabetes and cardiovascular disease interventions by community pharmacists: a systematic review. Ann Pharmacother. 2011;45(5):615-628.

16. Kozma CM, Reeder CE, Schulz RM. Economic, clinical, and humanistic outcomes: a planning model for pharmacoeconomic research. Clin Ther. 1993;15(6):1121-1132.

17. Rubin RR, Peyrot M. Quality of life and diabetes. Diabetes Metab Res Rev. 1999;15(3):205-218.

18. Rubin RR. Diabetes and quality of life. Diabetes Spectr. 2000;13(1): 21-23.

19. What quality of life? The WHOQOL Group. World Health Organization Quality of Life Assessment. World Health Forum. 1996;17(4): 354-356.

20. Guyatt GH, Feeny DH, Patrick DL. Measuring health-related quality of life. Ann Intern Med. 1993;118(8):622-629.

21. Bhatt JK, Thomas S, Nanjan MJ. Health outcome measures for diabetes mellitus: a review. Appl Res Qual Life. 2012;7:413-443.

22. El Achhab Y, Nejjari C, Chikri M, Lyoussi B. Disease-specific healthrelated quality of life instruments among adults diabetic: a systematic review. Diabetes Res Clin Pract. 2008;80(2):171-184. 
23. Speight J, Reaney MD, Barnard KD. Not all roads lead to Rome a review of quality of life measurement in adults with diabetes. Diabet Med. 2009;26(4):315-327.

24. The DCCT Research Group. Reliability and validity of a diabetes quality-of-life measure for the diabetes control and complications trial (DCCT). Diabetes Care. 1988;11(9):725-732

25. Bradley C, Todd C, Gorton T, Symonds E, Martin A, Plowright R. The development of an individualized questionnaire measure of perceived impact of diabetes on quality of life: the ADDQoL. Qual Life Res. 1999;8(1-2):79-91.

26. The World Health Organization Quality of Life Assessment (WHOQOL): development and general psychometric properties. Soc Sci Med. 1998;46(12):1569-1585.

27. Ware JE Jr, Sherbourne CD. The MOS 36-item short-form health survey (SF-36). I. Conceptual framework and item selection. Med Care. 1992;30(6):473-483.

28. Contopoulos-Ioannidis DG, Karvouni A, Kouri I, Ioannidis JPA. Reporting and interpretation of SF-36 outcomes in randomised trials: systematic review. BMJ. 2009;338:a3006.

29. Myers C, Wilks D. Comparison of Euroqol EQ-5D and SF-36 in patients with chronic fatigue syndrome. Qual Life Res. 1999;8(1-2):9-16.

30. van de Willige G, Wiersma D, Nienhuis FJ, Jenner JA. Changes in quality of life in chronic psychiatric patients: a comparison between EuroQol (EQ-5D) and WHOQoL. Qual Life Res. 2005;14(2):441-451.

31. Kheir NM, van Mil JW, Shaw JP, Sheridan JL. Health-related quality of life measurement in pharmaceutical care. Targeting an outcome that matters. Pharm World Sci. 2004;26(3):125-128.

32. Downs SH, Black N. The feasibility of creating a checklist for the assessment of the methodological quality both of randomised and nonrandomised studies of health care interventions. J Epidemiol Community Health. 1998;52(6):377-384.

33. Armour CL, Taylor SJ, Hourihan F, Smith C, Krass I. Implementation and evaluation of Australian pharmacists' diabetes care services. $J \mathrm{Am}$ Pharm Assoc (2003). 2004;44(4):455-466.

34. Clifford RM, Batty KT, Davis TME, et al. A randomised controlled trial of a pharmaceutical care programme for high-risk diabetic patients in an outpatient clinic. Int J Pharm Pract. 2002;10(2):85-89.

35. Krass I, Armour CL, Mitchell B, et al. The Pharmacy Diabetes Care Program: assessment of a community pharmacy diabetes service model in Australia. Diabet Med. 2007;24(6):677-683.

36. Krass I, Mitchell B, Song YJ, et al. Diabetes Medication Assistance Service Stage 1: impact and sustainability of glycaemic and lipids control in patients with type 2 diabetes. Diabet Med. 2011;28(8):987-993.

37. Balisa-Rocha BJ, Guimaraues VG, Mesquita AR, Aguiar PM, Krass I, Pereira de Lyra D Jr. Enhancing health care for type 2 diabetes in Northen Brazil: a pilot study of pharmaceutical care in community pharmacy. Afr J Pharm Pharmacol. 2012;6(35):2584-2591.

38. Correr CJ, Pontarolo R, Souza R, Venson R, Melchiors AC, Wiens A. Effect of a pharmaceutical care program on quality of life and satisfaction with pharmacy services in patients with type 2 diabetes mellitus. Braz J Pharm Sci. 2009;45(4):809-817.

39. Guirguis LM, Johnson JA, Farris KB, Tsuyuki RT, Toth EL. A pilot study to evaluate the impact of pharmacists as certified diabetes educators on the clinical and humanistic outcomes of people with diabetes. Can J Diabetes Care. 2001;25(4):266-276.

40. Arun KP, Murugan R, Kanna MR, Rajalakshmi S, Kalaiselvi R, Komathi V. The impact of pharmaceutical care on the clinical outcome of diabetes mellitus among a rural patient population. Int J Diabetes Dev Ctries. 2008;28(1):15-18.

41. Sriram S, Chack LE, Ramasamy R, Ghasemi A, Ravi TK, Sabzghabaee AM. Impact of pharmaceutical care on quality of life in patients with type 2 diabetes mellitus. J Res Med Sci. 2011;16(Suppl 1): S412-S418.

42. Abduelkarem A, Sackville M. Changes of some health indicators in patients with type 2 diabetes: a prospective study in three community pharmacies in Sharjah, United Arab Emirates. Libyan J Med. 2009;4(1):31-36.
43. Al Mazroui NR, Kamal MM, Ghabash NM, Yacout TA, Kole PL, McElnay JC. Influence of pharmaceutical care on health outcomes in patients with type 2 diabetes mellitus. $\mathrm{Br} \mathrm{J} \mathrm{Clin} \mathrm{Pharmacol.}$ 2009;67(5):547-557.

44. Ali M, Schifano F, Robinson P, et al. Impact of community pharmacy diabetes monitoring and education programme on diabetes management: a randomized controlled study. Diabet Med. 2012;29(9): e326-e333.

45. Baran RW, Crumlish K, Patterson H, et al. Improving outcomes of community-dwelling older patients with diabetes through pharmacist counseling. Am J Health Syst Pharm. 1999;56(15):1535-1539.

46. Cranor CW, Christensen DB. The Asheville Project: short-term outcomes of a community pharmacy diabetes care program. J Am Pharm Assoc (Wash). 2003;43(2):149-159.

47. Jaber LA, Halapy H, Fernet M, Tummalapalli S, Diwakaran H. Evaluation of a pharmaceutical care model on diabetes management. Ann Pharmacother. 1996;30(3):238-243.

48. Johnson CL, Nicholas A, Divine H, Perrier DG, Blumenschein K, Steinke DT. Outcomes from DiabetesCARE: a pharmacist-provided diabetes management service. J Am Pharm Assoc (2003). 2008;48(6): $722-730$.

49. Scott DM, Boyd ST, Stephan M, Augustine SC, Reardon TP. Outcomes of pharmacist-managed diabetes care services in a community health center. Am J Health Syst Pharm. 2006;63(21):2116-2122.

50. Cranor CW, Bunting BA, Christensen DB. The Asheville Project: long-term clinical and economic outcomes of a community pharmacy diabetes care program. J Am Pharm Assoc (Wash). 2003;43(2): 173-184.

51. Taylor SJ, Milanova T, Hourihan F, Krass I, Coleman C, Armour CL. A cost-effectiveness analysis of a community pharmacist-initiated disease state management service for type 2 diabetes mellitus. Int J Pharm Pract. 2001;13(1):33-40.

52. Krass I, Armour CL, Clarke P, et al. Pharmacy Diabetes Care Program. Available from: http://www.guild.org.au/iwov-resources/ documents/The_Guild/PDFs/CPA $\% 20$ and $\% 20$ Programs/3CPA $\% 20$ General/2002-518/2002-518_fr.pdf. Accessed December 16, 2012.

53. Vermeire E, Wens J, Van Royen P, Biot Y, Hearnshaw H, Lindenmeyer A. Interventions for improving adherence to treatment recommendations in people with type 2 diabetes mellitus. Cochrane Database Syst Rev. 2005;2:CD003638.

54. Egginton JS, Ridgeway JL, Shah ND, et al. Care management for type 2 diabetes in the United States: a systematic review and meta-analysis. BMC Health Serv Res. 2012;12:72.

55. Health Quality Ontario. Community-based care for the management of type 2 diabetes: an evidence-based analysis. Ont Health Technol Assess Ser. 2009;9(23):1-40.

56. Choe HM, Mitrovich S, Dubay D, Hayward RA, Krein SL, Vijan S. Proactive case management of high-risk patients with type 2 diabetes mellitus by a clinical pharmacist: a randomized controlled trial. Am J Manag Care. 2005;11(4):253-260.

57. Testa MA, Simonson DC. Health economic benefits and quality of life during improved glycemic control in patients with type 2 diabetes mellitus: a randomized, controlled, double-blind trial. JAMA. 1998;280(17):1490-1496.

58. Anderson RT, Narayan KM, Feeney P, et al. Effect of intensive glycemic lowering on health-related quality of life in type 2 diabetes: ACCORD trial. Diabetes Care. 2011;34(4):807-812.

59. Delamater AM, Jacobson AM, Anderson B, et al. Psychosocial therapies in diabetes: report of the Psychosocial Therapies Working Group. Diabetes Care. 2001;24(7):1286-1292.

60. Brown GC, Brown MM, Sharma S, Brown H, Gozum M, Denton P. Quality of life associated with diabetes mellitus in an adult population. J Diabetes Complications. 2000;14(1):18-24.

61. Burroughs TE, Desikan R, Waterman BM, Gilin D, McGill J. Development and validation of the diabetes quality of life brief clinical inventory. Diabetes Spectr. 2004;17:41-49. 
62. Horne R, Weinman J, Hankins M. The beliefs about medicines questionnaire: the development and evaluation of a new method for assessing the cognitive representation of medication. Psychol Health. 1999; 10:135-140.

63. Horne R, Hankins M, Jenkins R. The Satisfaction with Information about Medicienes Scale (SIMS): a new measurement tool for audit and research. Qual Health Care. 2001;10(3):135-140.

64. Bradley C. The 12-Item Well-Being Questionnaire: origins, current stage of development, and availability. Diabetes Care. 2000;23(6):875.

65. Svarstad BL, Chewning BA, Sleath BL, Claesson C. The Breif Medication Questionnaire: a tool for screening patient adherence and barriers to adherence. Patient Educ Couns. Jun 1999;37(2):113-124.

66. Pharmaceutical Care Rsearch Group, University of Granada (Spain). Pharmacotherapy follow-up: the Dader method (3rd revision: 2005). Pharmacy Practice. 2006;4(1):44-53.

67. Correr CJ, Pontarolo R, Melchiors AC, Rossignoli P, Fernandez-Llimos F, Radominski RB. [Translation to portuguese and validation of the Diabetes Quality Of Life Measure (DQOL-Brazil;)]. Arq Bras Endocrinol Metabol. 2008;52(3):515-522.

68. Jenkinson C, Layte R. Development and testing of the UK SF-12 (short form health survey). J Health Serv Res Policy. 1997;2(1):14-18.

69. MacKeigan LD, Larson LN. Development and validation of an instrument to measure patient satisfaction with pharmacy services. Med Care. 1989;27(5):522-536.
70. Johson JA. Coons SJ, Hays RD, Pickard AS. Health status and satisfaction with pharmacy services. Am J Manag Care. Feb 1999;5(2):163-170.

71. Health status questionnaire 2.0 user guide. Bloomington, MN: Health Outcome Institute; 1993.

72. The EuroQol Group. EuroQol—A new facility for the measurement of health related quality of life. Health Policy. 1990;16:199-208.

73. Bradley C, Plowright R, Stewart J, Valentine J, Witthaus E. The Diabetes Treatment Satisfaction Questionnaire change version (DTSQc) evaluated in insulin glargine trials shows greater responsiveness to improvements than the original DTSQ. Health Qual Life Outcomes. 2007:5:57.

74. Toobert DJ, Hampson SE, Glasgow RE. The summary of diabetes self-care activities measure: results from 7 studies and a revised scale. Diabetes Care. 2000;23(7):943-950.

75. Glasgow RE, Barrera M, Mckay HG, Boles SM. Social support, self-management, and quality of life among participants in an internet-based diabetes support program. Cyber Psychol and Behav. 1999;2:271-281.

76. Stewart AL, Hays RD, Ware JE, Jr. The MOS short-form general health survey. Reliability and validity in a patient population. Med Care. 1988;26(7):724-735
Clinical Audit

\section{Publish your work in this journal}

Clinical Audit is an international, peer-reviewed, open access journal focusing on the processes and outcomes of clinical audit in any area of healthcare. All aspects of patient care are addressed within the journal and practitioners from all disciplines are invited to submit their work. Areas covered include: Publication of audits; How an audit has changed practice;

\section{Dovepress}

Practical tips on how to do audits and to avoid pitfalls; How audits have changed patient care; Calls and justifications for new audits. The manuscript management system is completely online and includes a very quick and fair peer-review system, which is all easy to use. Visit http://www.dovepress. com/testimonials.php to read real quotes from published authors. 